make it alright). Large institutions should renew their commitment to promoting tolerance and harmony among society's diverse cultures.

The article by Hickling \& Hutchinson should be retracted immediately, and apologies offered by the publishers.

OLU ISAWUNMI, Department of Anaesthesia, John Radcliffe Hospital, Headley Way, Headington, Oxford OX3 9DU

\section{Reliability and validity of HoNOS}

Sir: We looked in vain for evidence of the statement by Chaplin \& Perkins (Psychiatric Bulletin. January 1999, 23, 20-21) that their study had assessed the reliability and validity of the pre-final version of the Health of the Nation Outcome Scales (HoNOS). The first claim relates to a comparison of the scores of 32 (out of 248) patients interviewed either by a psychologist. psychiatrist or by a nurse. What they call a lack of reliability seems to be large difference in the man total scores ( 15.6 and 6.4 respectively). In a second comparison involving only eight patients, similar mean total scores (13.75 and 14.25) were obtained by nurses rating independently of each other. It is impossible to interpret these figures without knowing, in substantial detail, how the study was carried out.

In the equivalent study of the pre-final HoNOS during the field trials (further details available from the authors upon request), there was a small by significant difference between nurses $(n=399)$ and psychiatrists $(n=60)$, probably reflecting differences between the settings (acute longer term and community) where the ratings took place there was a much larger difference between clinicians and social workers, which appeared to be associated with different rating thresholds, indicating as other studies have done a problem of calibration between professions.

So far as we can tell, no trial of 'validity' was carried out by the authors.

A further incidental but important point relates to terminology. The formulation "HoNOS rated half with hallucinations..." is inadmissible. HoNOS is not a person. The clinicians rated HoNOS. We do strongly agree with the recommendation that training should be supplemented by supervision, as emphasised in the HoNOS documentation.

JOHN WING, Professor of Psychiatry: and PAUL LELLIOTT, Director of College Research Unit, Royal College of Psychiatrists, 11 Grosvenor Crescent, London SWIX 7EE
Sir: In response to the correspondence from Wing \& Lelliott. I would like to make the following comments. HoNOS ratings were introduced as a routine measure of outcome in our service in 1995. An evaluation of their utility was considered necessary in order to assess their ability to measure change in our service which solely consists of people with severe and enduring psychiatric disability. Two senior staff members attended a training day organised by the Royal College of Psychiatrists and then trained all other senior team members. These senior professionals then trained professionals in all other areas of the service in the use of the scales and supervised the completion of the initial forms.

The scales were tested for reliability in only a minority of the patients as Wing \& Lelliott commented. To do so on a larger group would not have been possible without additional funding. Ratings by different professionals were all made in the same setting (long-term) so could not explain the differences. The study did not explicitly test validity as Wing \& Lelliott rightly state. However, some of the results were very surprising. The scales recorded zero on $6 \%$ of the patients which suggested a total absence of disability. In this group, the patients were reassessed by senior professionals who knew them well to confirm the clinical impression that they were indeed significantly disabled. This suggested that in this small group of patients that HoNOS lacked face validity. The most important finding of the study was that the different disciplines may have extremely different rating styles, a potential problem which can be addressed by multi-disciplinary group ratings.

R. H. Chaplin, Consultant Psychiatrist, Pathfinder Mental Health Service, Tooting and Furzedown Community Mental Health Team. Springfield Hospital, Tooting, London SW17 7DJ

\section{Police management of dangerous patients}

Your special article 'Police training for the management of dangerous patients' (Psychiatric Bulletin. January 1999, 23, 46-48) raises a number of questions. National Schizophrenia Fellowship members recognise that police are in the front-line, not through choice but as a result of the resource and planning failing associated with care in the community. They remain the only service that can be relied upon to turn up at any time of the day or night when called.

However, the Police Complaints Authority and Metropolitan Police Commissioner Sir Paul Condon, among others, now recognise what the National Schizophrenia Fellowship has been saying for years, that police training in dealing 